\title{
Advances in Biomedical Research: A Commentary
}

\author{
*Professor Jeffrey E. Jarrett \\ University of Rhode Island, USA
}

Received: September 19, 2017; Published: September 25, 2017

*Corresponding author: Professor Jeffrey E. Jarrett, PHD, Professor, University of Rhode Island, Kingston, RI (USA), Email: Jejarrett133@gmail.com

\section{Introduction to Comment}

Biomedical research is now a field not only opens to the gathering of medical data on diagnostic tests, epidemic research and disease prevention and cure. The purpose of biomedical research is now properly changed to the application modern methods of scientific sophistication whereby operations research, statistical analytics and analysis and data management often referred to as "Data Science" and for marketing purposes "Big Data."Research methods are now everywhere one sees from the production of most automotive parts to the check-out lines at most supermarkets and the design of seating arrangement to maximize cash inflow on aircraft. Not all satisfy all users of technological improvement but they are everywhere to satisfy the desires of the decision makers whose goals vary but often provide satisfaction to them. In the medical sciences, we observe trends in the direction of great changes in patient care and quicker methods for decision purposes. We often refer to this as automation but it is the advances in computer technologies that drive this mechanization of seemingly simple but technological advanced tasks to streamline patient care methodologies. The growth of these technologies in the future will speed up by breakthroughs in artificial intelligence which will continue the mechanization of tasks improve the quality of disease control, production of new pharmaceuticals, intensive care improvement, disease and epidemic prevention and water pollution and treatments after horrific storms which bring problems to keeping water pure, available and easy to transport to areas having great difficulties handling the aftermath of destructive environment conditions. Artificial Intelligence (AI) includes the leadership in Forensic Science Laboratories; Forensic Laboratory Key Business Metrics and Cost-Benefit Analyses; Laboratory Excellence and Ethics: An Essential Association; Laboratory Excellence and Ethics: An Essential Association; ISO Accreditation Implementation: A Framework to Implement a Quality and Writing Policies and Procedures.

\section{A Simple Example of Pharmaceutical Testing}

New pharmaceutical testing is a subject matter where a new cure for a disease is compared with two sample independently drawn where one group is given the experimental drug and second sample is given a placebo, a substance containing no medicinal value. Scientific testing requires the researcher to determine if there is a statistically scientific difference in the result of the experiment and furthermore the experiment drug produces better result and often how much better. Medical controls are often implement in order that the two independently drawn samples differ only with respect to whether the sample differs only by which pill is taken by the patient. The problem is whether the sample are large enough to draw scientific conclusions or the groups are dissimilar in other ways that can affect the final curative results. Furthermore, more scientific and sophisticated analyses could be employed that explain real differences when they occur. Multivariate statistical methods could be employed to examine the results which would lead analysts to quicker and better conclusion. In addition, data science and AI will produce the same results without human interference which enable much faster certainty in the results and bring new drugs to market at an exponentially faster pace.

\section{Conclusion}

The previous example and discussion will lead to great benefits for society. The above commentary leads to medical researchers rethinking their methodology, goal and purpose. The blending of intelligent (AI) software and comprehensive data analytics will eventually move health care analysts from the task of interpreting results to have protocols produced for them. Intelligent software will blend seamlessly with a decision maker's operations insights and produce a unique domain expertise to create better analytical conclusions in the world we live in and certainly after horrendous climatic events. 


\begin{tabular}{|l} 
BIOMEDICAL \\
RESEARCHES
\end{tabular}$\quad \begin{aligned} & \text { Assets of Publishing with us } \\
& \text { - Global archiving of articles }\end{aligned}$

